\title{
Introduction to the Special Issue on Physics of Societies
}

\author{
Arnab Chatterjee* \\ Condensed Matter Physics Division \\ Saha Institute of Nuclear Physics \\ 1/AF Bidhannagar, Kolkata 700064, India \\ arnabchat@gmail.com \\ Gul Unal Coban \\ DokuzEylul University, Buca/Izmir, Turkey \\ gulunalcoban@gmail.com \\ Ophir Flomenbom \\ Flomenbom-BPS Ltd, 19 Louis Marshal Tel Aviv 62668, Israel \\ ophir1974@flomenbom.net \\ Received 4 February 2017 \\ Revised 7 February 2017 \\ Published 4 April 2017
}

\begin{abstract}
How can we solve problems in the society that cause socio economic crises such as the one of 2008? Here we introduce the subject of this special issue, 'physics of societies', dealing with a general introduction to the subject, and the particular special issue projects' presentation, explaining why this subject is important to the scientific community and to the society, what are the problems that we can deal better using these approaches, and what can be suggested to do next in practice. We emphasize the strength of physics of socio-economic systems to treat problems in nations that are not solved with other approaches, and that these results can indeed affect the people, in particular with intervention schemes that support creating managed organizations, including support from socio-economic-organizations, for instance.
\end{abstract}

Keywords: Socio-physics; econo-physics; socio-econo-physics; biophysical economics; economic biophysics; physics of society; physiocrats; fairness in nations, Gini, opinion models, minority game, social modeling, civil society organisations.

PACS: 89.65.-s, 89.65.Gh, 89.65.-s.

*Present address: TCS Research, New Delhi, India

This is an Open Access article published by World Scientific Publishing Company. It is distributed under the terms of the Creative Commons Attribution 4.0 (CC-BY) License. Further distribution of this work is permitted, provided the original work is properly cited. 


\section{Introduction}

In this special issue, ${ }^{1-11}$ we deal with the subject termed as physics of societies, trying to summarize the field ${ }^{12-57}$ and to show where we can do better than other approaches to treat unsolved issues in societies.

Sociology deals with social groups and the individuals that built up the society, where the individuals' collective effect creates the culture they live in. In the cultural and institutional manner, occurring sets of pressures and forces determine the flow and directions of the people dynamics, opportunities, the politics, the economics, the wealth distribution's structure, etc.

In particular, we talk about the structure of the society, that is defined as an aggregate of (usually human) entities living together as a community, with persistent interactions, usually subjected to a common political authority and influenced by dominant cultural expectations. The relationship between individuals play a crucial role, and the emergent behavior helps individuals to benefit from it as a whole. The above idea can be extrapolated to economies, businesses and organizations, where the type of the interactions differs and the entities, their goals, behaviors, abilities, and other properties vary. Philosophy and science in general have had contributed to the understanding of the organization that takes place in the above, through years of rigorous formulation of the disciplines of economics, sociology and social sciences in general, the failure to understand several important phenomena has pointed at the apparent failure from the existing social and economic theories. Their failure can be explained by the methodology they used which is unlike the methodologies of mathematics and physical sciences that work with definitions of principles to predict and inference future of the facts, or yet with the complexity of people behavior and the power structure in nations. ${ }^{54,55}$ Collins $^{56}$ claims that it is because sociology has to deal with all kinds of difficulty relative to these sciences, in addition to the right of the subjects to change their actions, makes a difference. Experiencing the living world, individual choices are made and creating the socially collective, constantly changing the system. Among the important examples being the inability to predict financial market crashes and recessions, the accurate prediction of business failure, failure in crime management, failure in creating a stable structure in nations, difficulties in balancing the elite power, and many other matters that involve injustice.

What are the problems that prevent proper solutions to these matters? Can we explain in a better way nations with approaches from physics, biophysics, and other physical sciences and solve untreated socio-economic problems? In this special issue, we try to touch on these matters. We are suggesting that socio-econo-physics can do better than other approaches and give good examples and solutions to the unsolved problems or problems that are ignored. In this special issue,

(i) we deal with wealth densities modeling in Refs. 1, 3 and 4.

(ii) we present the case where socio-economic organizations can affect uniquely on society in Ref. 5 . 
(iii) In Ref. 6, a network model on youth violence and solutions are being presented.

(iv) A review about the source of economics with the physiocrats work in the $1750 \mathrm{~s}$ and what was developed from their approaches in the view of socio-econophysics are being discussed in Ref. 7 .

(v) In Ref. 8, the development of employment modeling is being discussed.

(vi) The discovery of patterns in the financial markets in China is being presented in Ref. 9.

(vii) Reference 10 talks about the influence of politics on science.

(viii) In Ref. 11 the conflicts statistics are being discussed.

In part II, general issues about physics of societies are being presented. Projects with various broad comments are presented in part III.

This special issue put forward a wide variety of work focusing on the (presented work) approaches' strength (relative to other approaches) to create better societies. The area of physics of societies can affect the citizens more and at a faster rate. We will continue to publish and deal with the areas mentioned, in Reports on Advances in Physical Sciences (RAPS).

\section{The Area of Socio-Econo-Physics}

Socio-economic systems seem to contain hidden patterns, similar to "laws" in natural systems, which are mostly robust under external interventions. Physicists have been adventurous in exploring socio-economic phenomena for a very long time, and the increased enthusiasm and activity in the last few decades have triggered the creation of the research area, involving physicists, computer scientists, mathematicians and social scientists, what we can define, "physics of societies" and socio-econo-physics, among various terms. Traditional or modern physics investigates the transformation of energy into motion, social physics examines the flow of thoughts and information into behaviors. There is a strong mathematical relation between the flow of thoughts and information and people's behavior. ${ }^{57}$ Therefore, life itself can be treated as a huge laboratory and which can be observed in its natural flow. For example, controlling traffic through locations monitored from mobile phones, monitoring diseases and epidemic dynamics, monitoring the consumption rate of a specific good, etc. This field has been developed with the availability of large scale data, predominantly electronic in nature, which are being increasingly exploited by statistical analysts and modeled with tools of statistical physics, biophysics, and mathematical biology among various areas. There is already an increasing demand from social scientists and economists to broaden their horizons ${ }^{12}$ to understand socio-economic phenomena better.

Many laws of nature are statistical in origin, and that is a well-established fact that applies across most areas of modern physics. This makes statistical physics a status of a core and stable discipline. The subject is developed into a very general framework and that makes it applicable to various areas outside the commonly 
perceived boundaries of physics, for e.g. biology, computer science and information technology, geosciences etc. It is not surprising that attempts to understand the nature of socio-economic phenomena with statistical physics has been underway for quite sometime, while the research activities are gaining prominence in the recent two decades. What is striking is that the strength of statistical physics to understand social systems has gone beyond being prominence. ${ }^{13-18}$ The fact that statistical physics treats its basic entities as particles, has an apparently similarity with society where the basic constituents are human individuals. In spite of the gross difference in the level of abstraction, human societies' features are similar to those widely observed in the physical systems. Transitions from order to disorder are observed and emergence of consensus on specific issues from a "configuration" with multiple options by converging to one or a few of them. Several measurable quantities in a society show scaling and universality. ${ }^{19}$ Dynamic socio-economic systems exhibit features like self-similarity ${ }^{20}$ while going from one phase to another, similar to that in critical phenomena observed in physical systems. These made many to believe that statistical physics can be used as a tool to effectively understand socio-economic systems. Yet this area of physics of societies is much richer and wider, including approaches from biology and biophysics which makes modeling complexity much more accurate, where we can zoom in and zoom out in the modeling of societies, describing general phenomena and their microscopic sources and reasoning.

Human social interactions are often seen to result in complex dynamics, resulting to create inequalities at various levels. Socio-economic inequalities ${ }^{1,2,7,21-25,54}$ basically are concern with the existence of unequal "wealth" and "fortunes" accumulated unjustly within the society. These usually contain structured and often recurrent patterns of unequal distributions of goods, wealth, rewards from biased opportunities, and biased punishments. Socio-economic inequalities are responsible for conflicts, wars, crisis, oppression, criminal activities, political instability and unrest, and they indirectly affect economic grow $\mathrm{th}^{26}$ in a region. Economic inequalities have been extensively studied in the context of income and wealth and opportunities, ${ }^{1,2,7,13,27,28,54}$ and also for energy consumption. ${ }^{29}$ The studies of inequality in society ${ }^{1,2,7,30-33,54}$ have always been very important, a topic of current focus and immediate global interest, in particular after the 2008 global economic crisis and that bringing researchers together across various disciplines to understand the inequality patterns, dynamics, and consequently socio-economic inequalities are quantified. The probability distributions of various quantities with the model that can create these provided the most detailed measures. What is usually observed is that most quantities display broad distributions, the most common are log-normals, powerlaws or their combinations. For example, the distribution of income is usually found to be exponential followed by a power law. ${ }^{1,13,34}$ However, such distributions can widely differ in their forms and subtleties, as such they are difficult to handle. The introduction of various indices like the Gini, ${ }^{35}$ Theil,${ }^{36}$ Pietra,,${ }^{37} \mathrm{k}$-index, ${ }^{38}$ and other socio-geometric indices, ${ }^{39,40,54}$ has helped to characterize various geometric features of these distributions and their sources. The latest Oxfam report showed ${ }^{41}$ that eight 
people own as much wealth as half of the world, and that is extremely alarming, and it is pointing at the rapidly increasing economic inequality across the globe. This is in continuation with the alarming facts raised by many during the whole of 20 th century, for e.g., in Ref. 42, yet also since a few years ago by Piketty and friends ${ }^{30}$ making a lot of appearances in the main stream media after the 2008 financial crisis.

\section{The Special Issue Projects and Various Other Comments}

In this issue, we deal with inequalities in the various papers. In "From the Physiocrats to Fairness in Nations", Adiguzel et al. ${ }^{7}$ present the initiation of economics (science of economics) with the work of the European Physiocrats in the 1750's, that treated the society's food production from agriculture as the major factor in describing the society. This approach has been improved to treat all sorts of computations about the energetic content, cost and value of that we create in nations, and then to the generalizations, including information flow and control, the power structure in nations, etc, the exact matters that are today forming the roots in the area of socio-econo-physics.

The "Gini of fairness" is presented in Ref. 1 and it also deals with inequalities, with the Gini of the income distribution (Gini is the number in the range zero and one that measures the departure of the distribution from the "all are equal" distribution). It develops the model to create wealth densities in societies, to derive the upper bound on the income density Gini in the fair society, where within the model and the various checked variants, that's $40 \%$. Ways to realize that are presented. Indeed, the first reference about the income density in society was due to Pareto who more than a century ago made extensive studies in Europe and found that the wealth distribution follows a power law tail for the richer section of the society, ${ }^{43}$ what we term now as Pareto law. Independently, Gibrat worked on the same problem and he proposed "a law of proportionate effect". ${ }^{44}$ Much later, Champernowne's systematic approach provided a probabilistic theory to justify Pareto's claim. ${ }^{45}$ Subsequent studies revealed that the distributions of income and wealth possess some globally stable and robust features (see, e.g., Ref. 13). In general, the bulk of the distribution of both income and wealth seems to reasonably fit both the log-normal and the Gamma distributions. Economists have a preference for the log-normal distribution, ${ }^{35,46}$ while statisticians ${ }^{47}$ and now, physicists ${ }^{48-50}$ prefer the Gamma distribution for the probability density or Gibbs' exponential distribution for the corresponding cumulative distribution. The high end of the distribution, known to be the "tail", is well described by a power law, as was founded by Pareto.

Nevertheless, the modeling of the society that results in these income distributions and the consequences from these models is a developing area of research, where the papers in this special issue, Refs. 1 and 2, can indeed show various intriguing results. Reference 1 developed a model with two groups, the society and the top $1 \%$, that interact and fight for wealth. The model showed that Gini beyond $40 \%$ is the value where the society is defined unfair, and ways to solve the problems are being 
suggested. The work from Ghosh and Biswas ${ }^{2}$ investigate how income and wealth distributions look like in a scenario where there is competition for resources, in the framework similar to the Minority Game. ${ }^{51,52}$ This study takes forward the zero-intelligence models setting for wealth distribution ${ }^{13}$ and empowers agents with strategies that depend on the action of the other agents.

Chandra and $\mathrm{Basu}^{3}$ wrote on the behavior of diffusion controlled opinion dynamics, where the agents execute simple exclusion process (SEP) on the ring and exchange opinion with neighboring agent according to a fixed rule. There, exists active-to-absorbing state phase transition depending on the lattice density of agents.

Crokidakis ${ }^{4}$ characterized the behavior of a three-state opinion model in the presence of noise that represents the independent behavior, the social temperature. The results suggested that the noise induces phase transitions at critical points that depended on the individuals' flexibility.

Financial markets have been the focus of the majority of the early works in 'econophysicss, ${ }^{53}$ with physicists working on to discover exact properties of the stock markets' fluctuations. Detailed analysis with physical tools and models have created the understanding of these characteristics, including the complex dynamics that the markets exhibit. However, the studies are far from being able to predict large fluctuations (crashes). A variety of approaches have been attempted in the last two decades. H.-L. Shi et al. ${ }^{9}$ reported on the time-varying return predictability of the Chinese Stock market. The market is observed by utilizing the various advanced statistical approaches.

Chatterjee and Chakrabarti ${ }^{11}$ research focused on the statistics of human deaths in man-made and natural disasters. They reported that the distribution of the number of human deaths in wars, battles, conflicts follow a broad distribution with a power law tail, similar to that in case of deaths in natural disasters like earthquakes, storms, wildfires, etc. They put forward a case where one can argue for a unified physical modeling for such processes.

Tao and $\mathrm{Wu}^{8}$ worked on social employment to find the Golden rule where employment will reach the best level at which every firm on average employs an optimal amount of workers. Cheong et al. ${ }^{6}$ worked on youth violence in Singapore. To address this complex social issue, they have developed a model based on the psychology, social science, and criminology literature. All these were put in a complex agent network model, where each complex agent is represented by a complex factor network of the 13 factors along with youth violence, coupled with each other through the extrinsic factors to form a complex social network. The sensitivity analysis on the model showed that the model is most sensitive to the parameters linking (1) nonintact family, (2) delinquency in general, (3) school disengagement, (4) peer delinquency, and (5) friends in gang-to-gang involvement. The intervention scenario simulations showed that it is critical to intervene early, and successful interventions work by tipping the balance between competing intrinsic and extrinsic factors. 
Kouneihe, and Barbachoux ${ }^{10}$ dealt with the effects on science from politics and businesses, calling for a management of the politics of the governance of the universities today, amongst other things.

The work from the socio economic organisation CESIE in Italy ${ }^{5}$ emphasized on how socio-economic-organisations form the platform to advance citizens' activities and to reshape the power structure in nations. The focus on social and online media showed the basic tool to create the influential activities to affect research and innovation and other civic matters, where particular examples where they work with the citizens are presented.

The idea of having a special issue on the physics of socio-economic systems is to put forward a wide variety of work in this area with the sub areas, focusing on contemporary topics of socio-economic relevance and strength (relative to other approaches) to create better societies. The special issue showed at least 4 approaches to treat societies,

(i) approaches from physics, econophysical analysis,

(ii) approaches from biophysics and mathematical biology, opinion modeling and the minority game,

(iii) general societal and economical approaches to present the issues with science and politics

(iv) approaches that explain how to perform things in the street in practice to restructure the nation's power structure, with the help from socio economic organizations.

This area of physics of societies can affect us a lot and fast, exactly where the bankbacked-biased-economics failed to help. This shows how much of scientific activities can have an effect on the society and the nation. We will continue to publish about that in RAPS and will organize conference in this area.

\section{References}

1. O. Flomenbom, The income Gini of fairness, Rept. Adv. Phys. Sci. 1(1) (2017) 1740004.

2. A. Ghosh and S. Biswas, Income and wealth distributions from stochastic strategy minority game, Rept. Adv. Phys. Sci. 1(1) (2017) 1740003.

3. A. K. Chandra and A. Basu, Diffusion controlled model of opinion dynamics, Rept. Adv. Phys. Sci. 1(1) (2017) 1740008.

4. N. Crokidakis, Nonequilibrium phase transitions induced by social temperature in kinetic exchange opinion models on regular lattices, Rept. Adv. Phys. Sci. 1(1) (2017) 1740001.

5. J. Mazaj, The case of civic society organization: Citizens' engagement into responsible research and innovation actions through the social media, Rept. Adv. Phys. Sci. 1(1) (2017) 1740010 .

6. S. A. Cheong et al., Youth violence and interventions: Insights from a complex agent network model, Rept. Adv. Phys. Sci. 1(1) (2017) 1740006.

7. Y. Adigüzel et al., From the physiocrats to fairness in nations, Rept. Adv. Phys. Sci. 1(1) (2017) 1750001. 
8. Y. Tao and X. Wu, Golden-rule level of the employment, Rept. Adv. Phys. Sci. 1(1) (2017) 1740005.

9. W.-X. Zhou, Time-varying return predictability in the Chinese stock markets, Rept. Adv. Phys. Sci. 1(1) (2017) 1740002.

10. J. Kouneihe and C. Barbachoux, The beginning of big sciences in physics: The price to pay, Rept. Adv. Phys. Sci. 1(1) (2017) 1740009.

11. A. Chatterjee and B. K. Chakrabarti, Fat tailed distributions for deaths in conflicts and disasters, Rept. Adv. Phys. Sci. 1(1) (2017) 1740007.

12. M. Buchanan, Economists must broaden their horizons, Nat. Phys. 13 (2017) 5.

13. B. K. Chakrabarti, A. Chakraborti, S. R. Chakravarty and A. Chatterjee, Econophysics of Income and Wealth Distributions (Cambridge University Press, Cambridge, 2013).

14. C. Castellano, S. Fortunato and V. Loreto, Statistical physics of social dynamics, Rev. Mod. Phys. 81 (2009) 591.

15. D. Stauffer, S. Moss De Oliveira, P. M. C. de Oliveira and J. S. S_ a Martins, Biology, Sociology, Geology by Computational Physicists (Elsevier, Amsterdam, 2006).

16. D. Stauffer, A biased review of sociophysics, J. Stat. Phys. 151(1-2) (2013).

17. S. Galam, Sociophysics: A Physicist's Modeling of Psycho-Political Phenomena (Springer, 2012).

18. P. Sen and B. K. Chakrabarti, Sociophysics: An Introduction (Oxford University Press, Oxford, 2013).

19. M. Buchanan, The Social Atom (Marshall Cavendish Business, London, UK, 2007).

20. S. Sinha, A. Chatterjee, A. Chakraborti and B. K. Chakrabarti, Econophysics: An Introduction (John Wiley \& Sons, Berin, 2010).

21. K. J. Arrow, S. Bowles and S. N. Durlauf, Meritocracy and Economic Inequality, (Princeton University Press, 2000).

22. J. E. Stiglitz, The Price of Inequality: How Today's Divided Society Endangers Our Future (WW Norton \& Company, 2012).

23. K. Neckerman, Social Inequality (Russell Sage Foundation, 2004).

24. J. H. Goldthorpe, Analysing social inequality: A critique of two recent contributions from economics and epidemiology, Eur. Sociological Rev. 26(6) (2010) 731.

25. A. Chatterjee, Socio-economic inequalities: A statistical physics perspective, In Econophysics and Data Driven Modelling of Market Dynamics, eds. F Abergel, H. Aoyama, B. K. Chakrabarti, A. Chakraborti, A. Ghosh (New Economic Windows, Springer, 2015), pp. $287-324$.

26. C. E. Hurst, Social Inequality: Forms, Causes and Consequences (Allyn and Bacon, Boston, 1995).

27. V. M. Yakovenko and J. Barkley Rosser Jr, Statistical mechanics of money, wealth and income, Rev. Mod. Phys. 81(4) (2009) 1703.

28. H. Aoyama, Y. Fujiwara and Y. Ikeda, Econophysics and Companies: Statistical Life and Death in Complex Business Networks (Cambridge University Press, Cambridge, 2010).

29. S. Lawrence, Q. Liu and V. M. Yakovenko, Global inequality in energy consumption from 1980 to 2010, Entropy 15(12) (2013) 5565.

30. T. Piketty and E. Saez, Inequality in the long run, Science 344(6186) (2014) 838.

31. A. Cho, Physicists say it's simple, Science 344(6186) (2014) 828.

32. G. Chin and E. Culotta, What the numbers tell us, Science 344(6186) (2014) 818.

33. Y. Xie, Undemocracy: Inequalities in science, Science 344(6186) (2014) 809.

34. A. A. Dragulescu and V. M. Yakovenko, Exponential and power-law probability distributions of wealth and income in the United Kingdom and the United States, Physica A 299(1) (2001) 213. 
35. C. Gini, Measurement of inequality of incomes, Econ. J. 31(121) (1921) 124.

36. H. Theil, Economics and Information Theory (North-Holland Amsterdam, 1967).

37. I. I. Eliazar and I. M. Sokolov, Measuring statistical heterogeneity: The pietra index, Physica A 389(1) (2010) 117.

38. A. Ghosh, N. Chattopadhyay and B. K. Chakrabarti, Inequality in societies, academic institutions and science journals: Gini and k-indices, Physica A 410(14) (2014) 30.

39. I. Eliazar, The sociogeometry of inequality: Part i, Physica A 426 (2015) 93.

40. I. Eliazar, The sociogeometry of inequality: Part ii, Physica A 426 (2015) 116.

41. Just 8 men own same wealth as half the world, https://www.oxfam.org/en/pressroom/ pressreleases/2017-01-16/just-8-men-own-same-wealt h-half-world, 16 January, 2017.

42. G. W. Domho, Who Rules America? Challenges to Corporate and Class Dominance (McGraw-Hill Humanities, NY, 2009).

43. V. Pareto, Cours d'economie politique, Rouge, Lausanne (1897).

44. R. Gibrat, Les in_egalit_es_economiques, Paris, Sirey (1931).

45. D. G. Champernowne and F. A. Cowell, Economic Inequality and Income Distribution (Cambridge University Press, Cambridge, 1998).

46. E. W. Montroll and M. F. Shlesinger, On 1/f noise and other distributions with long tails, Proc. Natl. Acad. Sci. 79 (1982) 3380.

47. R. V. Hogg, J. W. Mckean and A. T. Craig, Introduction to Mathematical Statistics (Pearson Education, Delhi, 2007).

48. A. Chatterjee, S. Yarlagadda and B. K. Chakrabarti, eds, Econophysics of Wealth Distributions (New Economic Windows Series, Springer-Verlag, Milan, 2005).

49. A. Chatterjee and B. K. Chakrabarti, Kinetic exchange models for income and wealth distributions, Eur. Phys. J. B60 (2007) 135.

50. V. M. Yakovenko and J. Barkley Rosser Jr, Colloquium: Statistical mechanics of money, wealth and income, Rev. Mod. Phys. 81 (2009) 1703.

51. D. Challet, M. Marsili and Y.-C. Zhang, Minority Games: Interacting Agents in Financial Markets (Oxford University Press, Oxford, 2004).

52. A. Chakraborti, D. Challet, A. Chatterjee, M. Marsili, Y.-C. Zhang and B. K. Chakrabarti, Statistical mechanics of competitive resource allocation using agent-based models, Phys. Rep. 552 (2015) 1.

53. R. N. Mantegna and H. E. Stanley, Introduction to Econophysics: Correlations and Complexity in Finance (Cambridge University Press, Cambridge, 2000).

54. O. Flomenbom, Biophys. Rev. Lett. 10 (2015) 157.

55. Here, the reader can search to seeing articles appearing in the media from familiar economists about the structure of $1 \%$ and $99 \%$ : P. Krugman, We are the $99.9 \%$ in NY Times (2011).

56. H. M. Collins, The sociology of scientific knowledge: Studies of contemporary science, Annual Review of Sociology 9 (1983) 265.

57. A. S. Pentdal, (2014), Retrieved from http://socialphysics.media.mit.edu/papers on March 2015. 